\title{
Ixekizumab for Treating Moderate-to-Severe Plaque Psoriasis: An Evidence Review Group Perspective of a NICE Single Technology Appraisal
}

\author{
Bram L. T. Ramaekers ${ }^{1}$ (D) Robert F. Wolff ${ }^{2} \cdot$ Xavier Pouwels $^{1} \cdot$ Marije Oosterhoff $^{1,3}$. \\ Anoukh Van Giessen ${ }^{1} \cdot$ Gill Worthy ${ }^{2} \cdot$ Caro Noake $^{2} \cdot$ Nigel Armstrong $^{2} \cdot$ \\ Jos Kleijnen $^{2,3} \cdot$ Manuela A. Joore ${ }^{1,3}$
}

Published online: 26 February 2018

(C) The Author(s) 2018. This article is an open access publication

\begin{abstract}
The National Institute for Health and Care Excellence invited Eli Lilly and Company Ltd, the company manufacturing ixekizumab (tradename Taltz ${ }^{\circledR}$ ), to submit evidence for the clinical and cost effectiveness of ixekizumab. Ixekizumab was compared with tumour necrosis factor- $\alpha$ inhibitors (etanercept, infliximab, adalimumab), ustekinumab, secukinumab, best supportive care and, if non-biological treatment or phototherapy is suitable, also compared with systemic non-biological therapies and phototherapy with ultraviolet B radiation for adults with moderate-to-severe plaque psoriasis. Kleijnen Systematic Reviews Ltd, in collaboration with Maastricht University Medical Center, was commissioned as the independent Evidence Review Group. This article presents a summary of the company submission, the Evidence Review Group report and the development of the National Institute for Health and Care Excellence guidance for the use of this drug in England and Wales by the Appraisal Committee. The Evidence Review Group produced a critical review of the clinical and cost effectiveness of ixekizumab based on the company submission. The company submission presented three randomised controlled trials identified in a systematic review. All randomised controlled trials were phase III, multicentre placebo-controlled trials including
\end{abstract}

Bram L. T. Ramaekers

bram.ramaekers@mumc.nl

1 Department of Clinical Epidemiology and Medical Technology Assessment (KEMTA), Maastricht University Medical Center, P.O. Box 5800, 6202 AZ Maastricht, The Netherlands

2 Kleijnen Systematic Reviews Ltd, York, UK

3 Care and Public Health Research Institute (CAPHRI), Maastricht University, Maastricht, The Netherlands
3866 participants with moderate-to-severe psoriasis. Two trials also included an active comparator (etanercept). All randomised controlled trials showed statistically significant increases in two primary outcomes, static Physician Global Assessment $(0,1)$ and improvement of $75 \%$ from baseline in the Psoriasis Area and Severity Index. Ixekizumab was generally well tolerated in the randomised controlled trials, with similar discontinuation rates because of adverse events as placebo or etanercept. The most frequent adverse events of special interest were infections and injection-site reactions. The company submission also included a network meta-analysis of relevant comparators. The Evidence Review Group highlighted some issues regarding the systematic review process and an issue with the generalisability of the findings in that the trials failed to include patients with moderate psoriasis according to a widely used definition. This issue was considered by the Appraisal Committee and the population was deemed generalisable to patients in England and Wales. Based on the network metaanalysis, the Appraisal Committee concluded that ixekizumab was more clinically effective than adalimumab and ustekinumab, and agreed it was likely that ixekizumab was similarly effective compared with secukinumab and infliximab while tolerability was similar to other biological treatments approved for treating psoriasis. The Evidence Review Group's critical assessment of the company's economic evaluation highlighted a number of concerns, including (1) the use of relative outcomes such as Psoriasis Area and Severity Index response to model the cost effectiveness; (2) the exclusion of the consequences of adverse events; (3) the assumption of no utility gain in the induction phase; (4) equal annual discontinuation rates for all treatments; (5) the selection of treatment sequences for consideration in the analyses and; (6) the transparency of the Visual Basic for Applications code used to develop the 
model. Although some of these issues were adjusted in the Evidence Review Group base case, the Evidence Review Group could not estimate the impact of all of these issues, and thus acknowledges that there are still uncertainties concerning the cost-effectiveness evidence. In the Evidence Review Group base-case incremental analysis, the treatment sequence incorporating ixekizumab in the second line has an incremental cost-effectiveness ratio of $£ 25,532$ per quality-adjusted life-year gained vs. the etanercept sequence. Ixekizumab in the first-line sequence has an incremental cost-effectiveness ratio of $£ 39,129$ per qualityadjusted life-year gained compared with the treatment sequence incorporating ixekizumab in the second line. Consistent with its conclusion regarding clinical effectiveness, the Appraisal Committee concluded that the cost effectiveness of ixekizumab for treating moderate-to-severe plaque psoriasis was similar to that of other biological treatments, already recommended in previous National Institute for Health and Care Excellence guidance. The committee concluded that the incremental cost-effectiveness ratio was within the range that could be considered a cost-effective use of National Health Service resources.

\section{Key Points for Decision Makers}

Ixekizumab showed a significantly significant improvement of clinically relevant outcomes in the treatment of moderate-to-severe plaque psoriasis. More adverse events occurred under ixekizumab than under placebo, most frequently infections and injection-site reactions.

Using relative outcomes, such as the Psoriasis Area and Severity Index, to construct the model structure might bias the cost-effectiveness estimates.

Transparency and documenting the technical implementation of the model are essential to facilitate model scrutiny by external and/or internal reviewers.

The National Institute for Health and Care Excellence Appraisal Committee has recommended ixekizumab within its marketing authorisation, as an option for treating moderate-to-severe plaque psoriasis.

\section{Introduction}

To be recommended by the National Institute for Health and Care Excellence (NICE) for use within the National Health Service (NHS), health technologies must be clinically effective and represent cost-effective use of NHS resources. Ixekizumab was appraised under the NICE single technology appraisal (STA) process, which typically considers new technologies within a single indication. Within the single technology appraisal process, the company (Eli Lilly and Company Ltd) provided NICE with a written submission, including an executable health economic model, detailing the company's estimates of the clinical and cost effectiveness of ixekizumab. The company's submission (CS) [1] was critically reviewed by the Evidence Review Group (ERG), an independent external organisation (Kleijnen Systematic Reviews), which produced an ERG report [2]. After consideration of the submitted evidence (the CS, ERG report, as well as testimony from stakeholders), the NICE Appraisal Committee (AC) issued the Final Appraisal Determination consisting of guidance regarding whether to recommend the technology, which is open to appeal [3].

\section{Decision Problem}

The population, according to the final scope issued by NICE, is defined as "adults with moderate to severe plaque psoriasis" [4]. In the CS, the population definition is narrower ("moderate to severe plaque psoriasis in adults who are candidates for systemic therapy") but appears to be in line with the final scope [1]. However, there is no agreed consensus on the terminology used to clarify the severity of psoriasis with various Psoriasis Area and Severity Index (PASI) thresholds [and Dermatology Life Quality Index (DLQI) restrictions] suggested to define moderate-to-severe or severe psoriasis, respectively.

Psoriasis is a common, chronic, genetic systemic inflammatory skin disease characterised by the appearance of red, thick and scaly plaques. Although psoriasis can appear on any part of the skin, it is most often found on the elbows, knees, scalp, legs, trunk and/or nails. Plaque psoriasis (hereafter psoriasis) represents the most common form of the disease and has a substantial impact on overall health; this includes systemic comorbidities such as depression, anxiety and suicidality in moderate-to-severe cases. The progression of the disease is unpredictable but generally follows a relapsing and remitting course impacting patients' quality of life.

The definition of the intervention in the CS [1] is in line with the definition in the final scope and identical to the 
definition used in the summary of product characteristics by the European Medicines Agency, which reads: "The recommended dose is $160 \mathrm{mg}$ by subcutaneous injection (two $80 \mathrm{mg}$ injections) at Week 0 , followed by $80 \mathrm{mg}$ (one injection) at Weeks 2, 4, 6, 8, 10, and 12, then maintenance dosing of $80 \mathrm{mg}$ (one injection) every 4 weeks" [5].

The scope issued by NICE lists six comparators [4]. Four comparators "for people with severe psoriasis for whom non-biologic systemic treatment or phototherapy is inadequately effective, not tolerated or contraindicated":

1. Tumour necrosis factor- $\alpha$ inhibitors (etanercept, infliximab, adalimumab).

2. Ustekinumab.

3. Secukinumab.

4. Best supportive care (BSC).

Two additional comparators are listed "if non-biologic treatment or phototherapy is suitable":

5. Systemic non-biological therapies (including acitretin, ciclosporin, fumaric acid esters, methotrexate).

6. Phototherapy with ultraviolet $\mathrm{B}$ radiation.

Some of these comparators were excluded in the CS as "there was insufficient evidence to include other non-biologic systemic therapies and phototherapy (i.e. acitretin, fumaric acid esters and phototherapy) that were listed in the scope" [2].

\section{Independent Evidence Review Group Review}

The sections below (3.1-3.4) summarise the evidence presented in the CS, as well as the ERG's review of that evidence.

\subsection{Clinical Effectiveness Evidence Submitted by the Company}

The CS conducted a systematic review to identify randomised controlled trials (RCTs) to form the evidence base for the clinical efficacy of ixekizumab in the treatment of moderate-to-severe psoriasis. Three RCTs were identified: UNCOVER-1, UNCOVER-2 and UNCOVER-3 $[6,7]$.

The UNCOVER studies were phase III, multicentre, randomised, double-blind, placebo-controlled, parallelgroup outpatient trials comparing the efficacy and safety of ixekizumab with placebo in patients with moderate-tosevere psoriasis. In addition, the UNCOVER-2 and UNCOVER-3 studies included an active comparator (etanercept) arm. In total, these RCTs included 3866 participants.

The primary outcomes were the severity of psoriasis after 12 weeks [static Physician Global Assessment $(0,1)$ ] and the response rate [improvement of $75 \%$ from baseline in the PASI (PASI 75)]. In all three UNCOVER trials, there were statistically significant increases in static Physician Global Assessment $(0,1)$ and PASI 75 response rates for patients treated with ixekizumab compared with placebo and etanercept at week 12 (Table 1). The improvements in PASI response rate appeared to be maintained for up to 60 weeks during the long-term extension period [2]. Compared with baseline, health-related quality of life improved in significantly more patients treated with ixekizumab than with placebo and etanercept (e.g. UNCOVER1, ixekizumab $80 \mathrm{mg}$ every 2 weeks vs. placebo: odds ratio 41.54 [ $95 \%$ confidence interval 25.37-68.02]). The relative performance of ixekizumab in difficult-to-treat areas, including nails, scalp and palmoplantar areas, was broadly better than placebo and etanercept. However, the improvement in psoriasis symptoms of the face which was included in the final scope issued by NICE was not reported in any of the UNCOVER studies.

Ixekizumab was generally well tolerated in the UNCOVER trials, with similar discontinuation rates owing to adverse events as placebo or etanercept. The most frequent adverse events of special interest observed in the UNCOVER studies were infections and injection-site reactions. Two deaths were recorded in the UNCOVER 1 trial (one by myocardial infarction and the other of unknown cause).

\subsubsection{Critique of Clinical Effectiveness Evidence and Interpretation}

When preparing the Final Appraisal Determination, the AC had access to results from three network meta-analyses (NMAs) including 31 studies comparing the relative efficacy of ixekizumab against a network of relevant comparators, including adalimumab, ciclosporin, etanercept, infliximab, methotrexate, secukinumab and ustekinumab. The results were marked as 'academic in confidence', i.e. are not in the public domain. The ERG identified another study [8], which should have been considered in these analyses. Including the additional study resulted in only small changes in PASI 75 at week 12. Overall, the ERG believes that it was appropriate to undertake the NMAs and the results obtained by the company were robust when compared with the results of the ERG analysis.

However, the ERG highlighted an issue with the generalisability of the findings as it seemed that the UNCOVER trials failed to include patients with moderate psoriasis according to a widely used definition. In the CS, moderate-to-severe psoriasis was defined as a total PASI score of 10 or more and a DLQI score of more than 10 . However, the patients recruited in the UNCOVER trails were those with PASI scores $\geq 12$ and no restriction related 


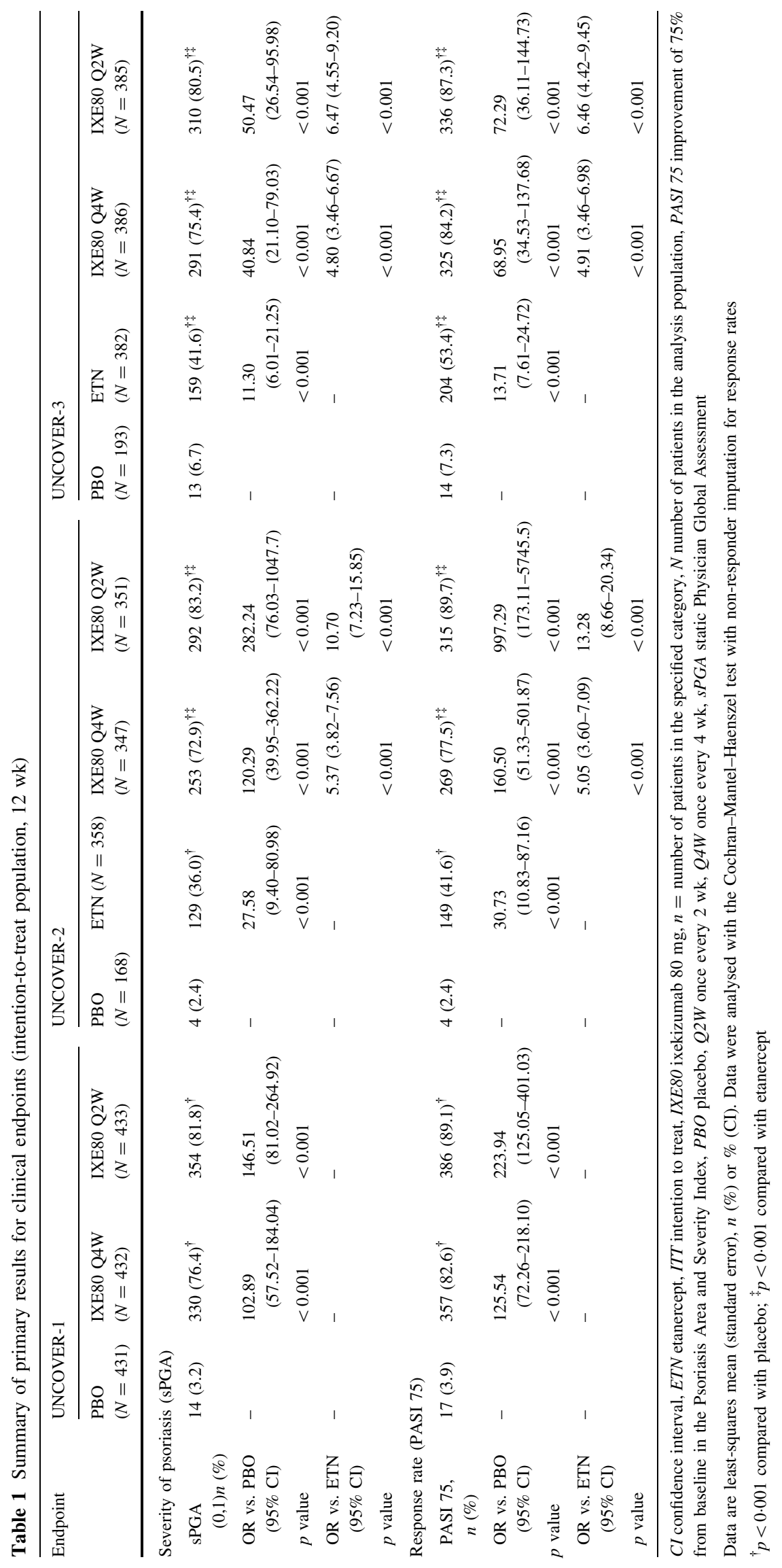


to DLQI. The ERG notes that there is no agreed consensus on diagnostic criteria or tests available to set a threshold between moderate and severe in current clinical guidelines. According to the clinical expert the ERG consulted, a PASI score of more than 10 (or 12) is used as the threshold for moderate/severe psoriasis combined when using systematic therapy rather than topical therapy.

Furthermore, the ERG identified a few issues in the systematic review process. It was unclear whether any language restrictions were used when searching for relevant literature. Similarly, the CS did not report sufficient information to determine whether the extracted data were assessed for accuracy. Ideally, the UNCOVER trials should have included some relevant measures to detect clinical improvement of facial psoriasis.

\subsection{Cost-Effectiveness Evidence Submitted by the Company}

A de novo Markov state-transition model (monthly cycle time) was developed in Visual Basic for Applications (VBA) with a Microsoft Excel interface. The economic evaluation used the perspective of the NHS. Utilities and costs were discounted at $3.5 \%$ over a lifetime time horizon. The model consists of four treatment-related health states: induction (trial) period, maintenance, BSC and death (Fig. 1). At the end of the induction period, PASI response categories are used to determine the utility gain experienced in the maintenance state. Patients who meet the minimum response criterion of PASI 75 in the CS base case (i.e. $75 \%$ reduction in the PASI score) continue treatment in the maintenance state. If patients do not have an adequate level of response, they enter another induction period upon initiating the next treatment line, either active treatment or BSC. Annual treatment discontinuation in the maintenance health state is assumed to be equal across all treatments.

The model structure (Fig. 1) was designed to compare treatment sequences (instead of individual treatments). Each treatment sequence considered in the model consists of three biological treatments followed by BSC (Table 2). The biological treatments included are: adalimumab, etanercept, ustekinumab, secukinumab and infliximab. The ordering of the biological treatments was based on market share, with the assumption that treatments are not repeated, and alternated in terms of mechanism of action. Ixekizumab was only modelled as a first-line treatment. The base-case economic evaluation considers biological-naïve patients who have not responded to prior conventional systemic therapies, and are eligible for biological therapies approved in the UK, i.e. as a first-line biological therapy.

The difference between treatment sequences is driven by a difference in PASI response (which determines the proportion of patients eligible for maintenance treatment, and

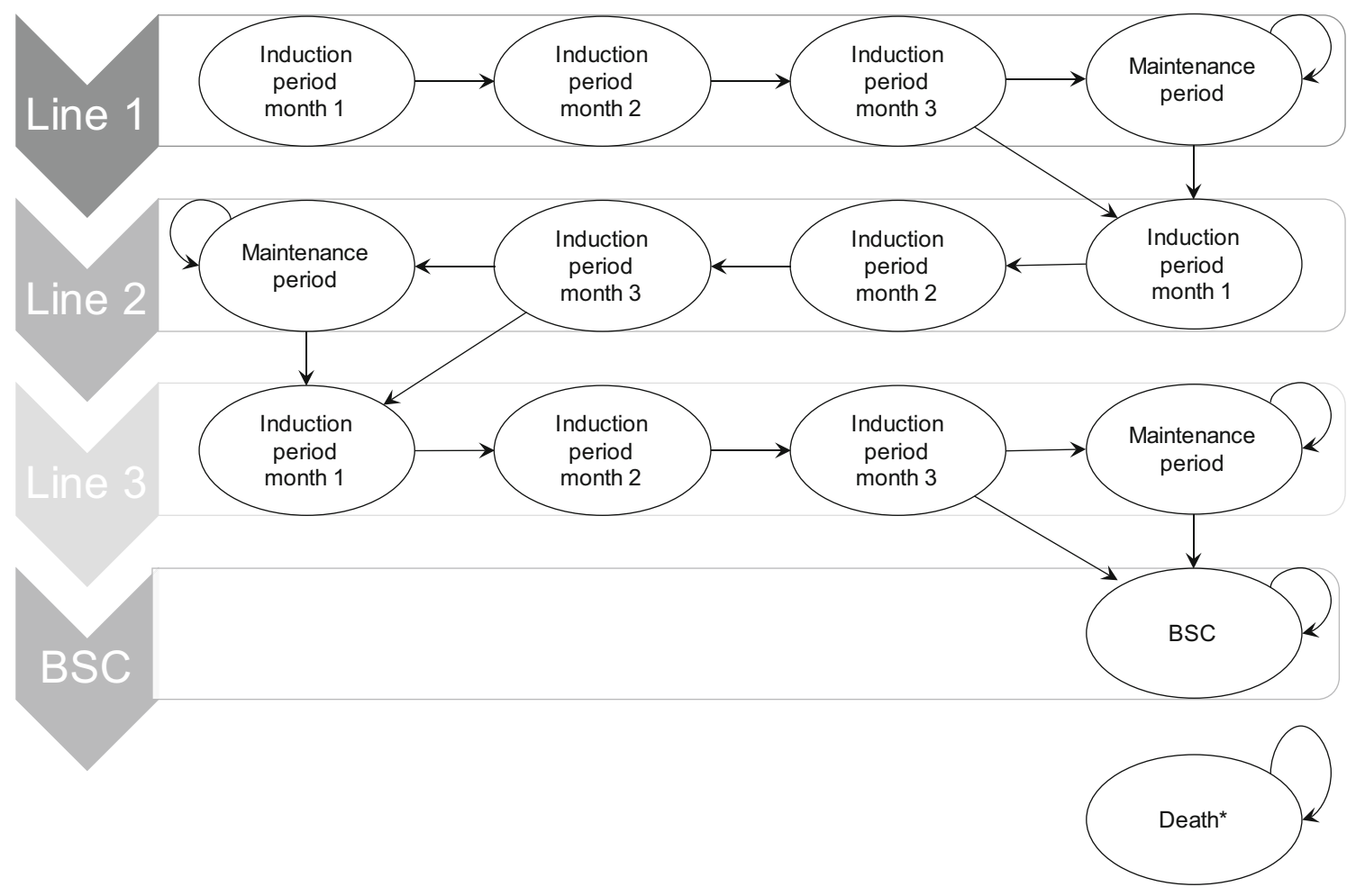

Fig. 1 Model structure. *Arrows to the death state from all other states are removed to simplify the figure. BSC best supportive care 
Table 2 Overview of treatment sequences considered in the company submission base case

\begin{tabular}{lllll}
\hline Sequence & First line & Second line & Third line & Fourth line \\
\hline A & Ixekizumab & Ustekinumab $90 \mathrm{mg}$ & Infliximab & BSC \\
$\mathrm{B}$ & Adalimumab & Ustekinumab $90 \mathrm{mg}$ & Infliximab & BSC \\
$\mathrm{C}$ & Etanercept $50 \mathrm{mg}$ & Ustekinumab $90 \mathrm{mg}$ & Infliximab & BSC \\
$\mathrm{D}$ & Infliximab & Ustekinumab $90 \mathrm{mg}$ & Adalimumab & BSC \\
$\mathrm{E}$ & Secukinumab & Ustekinumab $90 \mathrm{mg}$ & Infliximab & BSC \\
$\mathrm{F}$ & Ustekinumab $45 \mathrm{mg}$ & Adalimumab & Infliximab & BSC \\
$\mathrm{G}$ & Ustekinumab $90 \mathrm{mg}$ & Adalimumab & Infliximab & BSC \\
$\mathrm{H}^{\mathrm{a}}$ & Adalimumab & Ixekizumab & Infliximab & BSC \\
\hline
\end{tabular}

$B S C$ best supportive care

${ }^{a}$ This sequence was added by the Evidence Review Group hence utility gain and costs of treatment) and a difference in costs of single treatments. The PASI response for each single treatment was based on the absolute probabilities of achieving $a \geq 75 \%(\geq 50$ and $\geq 90 \%$ used in sensitivity analyses) reduction in PASI estimated in the NMA. The PASI response of BSC was based on the placebo groups in the trials included in the NMA. It is assumed that the PASI response of a treatment is not influenced by the position of the treatment in the treatment sequence.

Utility gains associated with a PASI response were estimated using regression analysis on EQ-5D-5L data obtained from a subset of patients with DLQI $>10$ at baseline in the UNCOVER trials. For all patients who discontinued the study before the end of the induction period (week 12), the last EQ-5D-5L value, if collected at the visit prior to discontinuation, was used as a proxy for the week 12 value using the last-observation-carried-forward method $[9,10]$. In the case that no previous postbaseline observations were available, missing values were not imputed.

The following healthcare costs were considered (cost year $=2016$ ): drug costs, drug administration costs, monitoring costs (during the induction and maintenance periods), non-responder costs and BSC costs. Adverse event costs were not considered in the base-case analysis but included in a scenario analysis (adverse events entailed malignancies and severe infections). Drug costs were mostly based on list prices, except for ustekinumab $90 \mathrm{mg}$. The biosimilar prices of etanercept and infliximab were used in the company base-case analysis. Best supportive care costs (applied after not responding to three biological treatments) were assumed to equal the healthcare costs incurred by a biological-naïve patient population.

As labelled by the company, base-case results (Table 3) were provided for biological-naïve patients with prior systemic non-response and moderate-to-severe psoriasis (PASI $\geq 10$ and DLQI $>10$ ). The incremental cost-effectiveness ratio (ICER) for the ixekizumab sequence vs. the etanercept sequence was $£ 32,541$ (probabilistic results) per quality-adjusted life-year (QALY) gained. Other treatment sequences were dominated (secukinumab sequence) or extendedly dominated by the ixekizumab sequence. The results of the probabilistic sensitivity analysis showed that the etanercept sequence and the ixekizumab sequence have the highest probabilities of being cost effective. The etanercept sequence was the most cost-effective treatment sequence up to a willingness-to-pay threshold of $£ 34,000$ per QALY gained. For a willingness-to-pay threshold above $£ 34,000$ per QALY gained, the ixekizumab sequence had the highest probability of cost effectiveness.

The most influential parameters in the deterministic sensitivity analyses of the ixekizumab vs. the etanercept sequence were drug costs, discount rates (both costs and QALYs) and the annual treatment discontinuation rate. In the deterministic sensitivity analyses of the ixekizumab vs. the secukinumab sequence, PASI 75 response rates for both ixekizumab and secukinumab were the most influential parameters.

\subsubsection{Critique of Cost-Effectiveness Evidence and Interpretation}

The ERG agrees that the treatment sequencing approach is superior to comparing single treatments. Apart from the treatment sequencing approach and modelling the 100\% PASI response as a separate category, the model structure is similar to models used in previous technology appraisals. Although common in this field, the ERG questioned the use of a relative PASI response to model the cost effectiveness as it may not reflect true differences in costs and healthrelated quality of life between treatments and treatment sequences. Regarding the model structure, the ERG also questioned the exclusion of the consequences of adverse events, the assumption of no utility gain in the induction phase and equal annual discontinuation rates for all treatments. Perspective, time horizon and discounting are in accordance with the NICE reference case. 
Table 3 Overview of incremental analyses for company submission (CS) base case and Evidence Review Group (ERG) base case (probabilistic results) Source: ERG report [2] Table 5.26

\begin{tabular}{|c|c|c|c|c|c|}
\hline Sequence & Total costs $(\mathfrak{f})$ & Total QALYs gained & Incremental costs $(£)$ & Incremental QALYs & ICER $(£)$ fully incremental \\
\hline \multicolumn{6}{|l|}{ CS base case } \\
\hline C: ETN sequence & 145,831 & 1.302 & - & - & - \\
\hline F: UST45 sequence & 149,493 & 1.341 & 3661 & 0.039 & Extendedly dominated \\
\hline B: ADA sequence & 149,587 & 1.354 & 3756 & 0.052 & Extendedly dominated \\
\hline G: UST90 sequence & 149,966 & 1.357 & 4134 & 0.055 & Extendedly dominated \\
\hline D: INF sequence & 151,894 & 1.362 & 6063 & 0.060 & Extendedly dominated \\
\hline A:IXE sequence & 151,972 & 1.491 & 6141 & 0.189 & $£ 32,541$ \\
\hline E: SEC sequence & 179,702 & 1.457 & 33,871 & 0.155 & Dominated \\
\hline \multicolumn{6}{|l|}{ ERG base case } \\
\hline C: ETN sequence & 147,438 & 1.345 & - & - & - \\
\hline H:ADA-IXE sequence & 150,574 & 1.468 & 3136 & 0.123 & 25,532 \\
\hline F: UST45 sequence & 151,103 & 1.389 & 3665 & 0.044 & Dominated \\
\hline B: ADA sequence & 151,311 & 1.405 & 3874 & 0.060 & Dominated \\
\hline G: UST90 sequence & 151,629 & 1.408 & 4191 & 0.063 & Dominated \\
\hline A: IXE sequence & 153,356 & 1.539 & 5918 & 0.194 & 39,129 \\
\hline D: INF sequence & 153,613 & 1.412 & 6175 & 0.066 & Dominated \\
\hline E: SEC sequence & 176,999 & 1.504 & 29,561 & 0.159 & Dominated \\
\hline
\end{tabular}

$A D A$ adalimumab, $E T N$ etanercept $50 \mathrm{mg}$, ICER incremental cost-effectiveness ratio, $I N F$ infliximab, $I X E$ ixekizumab, $Q A L Y s$ quality-adjusted life-years, SEC secukinumab, UST45 ustekinumab $45 \mathrm{mg}$, UST90 ustekinumab $90 \mathrm{mg}$

The population in the base-case analysis was labelled by the company as biological-naive patients with prior systemic non-response and moderate-to-severe psoriasis (PASI $\geq 10$ and DLQI $>10$ ). This is not fully in line with the scope, nor is it fully in line with the populations used to estimate values for input parameters. According to the ERG, the base-case analysis reflects a population for whom biological treatment is considered. Part of this population will be biological naïve and the majority of these patients will have not responded to prior systemic treatment. In the UNCOVER trials combined (used to estimate input parameters), $74 \%$ were biological naïve and only $36 \%$ of the patients had never used previous systemic therapies.

Although the ERG acknowledges that the submission could not possibly include all possible treatment sequences, the ERG thinks it is especially important to also consider a treatment sequence in which ixekizumab is a second-line treatment instead of a first-line treatment. According to the clinical expert consulted by the ERG, currently, clinicians would likely be inclined to use ixekizumab as a second line of therapy because more experience and safety data are available for tumour necrosis factor- $\alpha$ inhibitors and ustekinumab than for ixekizumab.

The PASI response was obtained from the NMA, and all usual caveats apply to the validity of comparative effectiveness estimates derived with this methodology. In addition, the ERG concludes that the populations included in the trials, considered in the NMA, may not fully reflect the population in the scope, as it was impossible to perform the NMA on patients with PASI $\geq 10$ and DLQI $>10$. The assumption that BSC after three lines of biological treatment equals placebo alongside a (mostly first-line) biological is questionable. It seems however plausible to assume that the treatment response to BSC in that setting (i.e. after failure to three biological therapies) will be very modest. It is debatable to assume that discontinuation is equal across all treatments, but reliable data to inform treatment specific discontinuation rates were lacking.

The ERG considered the utility estimates used by the company as uncertain for the following two reasons. First, one regression model was fitted, and alternative models were presented upon request. However, performance and diagnostic statistics were not provided. Hence, the ERG was unable to determine whether the model that was used to determine utility gain per PASI response category is the optimal one. Second, the ERG questions the use of the lastobservation-carried-forward method to impute values for patients who discontinued. Because of the number of patients this concerned and the reasons for discontinuation are unknown, the ERG was unable to assess the impact of this assumption. Additionally, although the ERG agrees with the use of the subset of patients with DLQI $>10$ at baseline from UNCOVER to estimate utility gain, as it describes the population in the scope better, the ERG is 
concerned about the inconsistency with the use of the total intention-to-treat population for calculating the PASI response.

In general, the ERG considers the costs as consistent with previous technology appraisals and adequate for the current decision problem. An area of concern is the costs of BSC. There is a lack of evidence on the costs of BSC in patients who have not responded to three biological therapies, which renders the estimate uncertain. In addition, the ERG identified errors in the CS base case, which were altered in the ERG base case (see below for more details).

\subsection{Additional Work Undertaken by the Evidence Review Group}

The ERG defined a new base case that included multiple adjustments to the original base case presented in the CS. The ERG fixed errors in the calculation of adverse event rates and costs, considered lower and upper quartiles of NHS reference costs to calculate standard errors for use in the PSA, corrected the number of secukinumab administrations during the maintenance period and used linear utility gains during the induction period instead of no gain during the induction period. In addition, the ERG added a treatment sequence, with ixekizumab as second-line therapy (ADA-IXE: Adalimumab $>$ ixekizumab $>$ biosimilar infliximab $>$ BSC). Adalimumab has been chosen as the first-line therapy in this sequence as it had the largest market share for the first-line therapy of patients with psoriasis in 2014 according to the company.

Fixing errors increased the costs of all comparators, and applying linear utility gain in the induction period increased QALYs for all treatment sequences. In the ERG base-case incremental analysis (Table 3), the ADA-IXE sequence has an ICER of $£ 25,532$ per QALY gained vs. the etanercept sequence, and the ixekizumab in the first-line sequence has an ICER of $£ 39,129$ per QALY gained compared with ADA-IXE (i.e. ixekizumab in the secondline sequence). The ADA-IXE sequence has a probability of being cost effective of $22.8 \%$ at a threshold of $£ 20,000$ per QALY gained, and $52.9 \%$ at a threshold of $£ 30,000$ per QALY gained. This is 2.8 and $13.2 \%$, respectively, for ixekizumab in the first-line sequence.

Additional exploratory sensitivity analyses were performed to examine the potential impact of various alternative assumptions. All exploratory analyses increased the ICER of the ixekizumab treatment sequence (in the full incremental analysis), except when the BSC costs were increased. In each fully incremental analysis, ADA-IXE was compared with the etanercept sequence, followed by ixekizumab as first-line therapy compared with ADA-IXE. All other comparators were (extendedly) dominated. The largest impact on the ICER was observed when using the intention-to-treat population from the UNCOVER trials to calculate utility gain per PASI response category. This increased the ICER of the ADA-IXE sequence vs. the etanercept sequence to $£ 36,314$ per QALY gained, and the ICER of ixekizumab in the first-line sequence vs. ADAIXE to $£ 55,243$ per QALY gained.

It should be noted that secukinumab is available in the NHS under a confidential patient access scheme price arrangement. Consequently, the analyses presented in the current report do not represent the true value for money of secukinumab.

\subsection{Conclusion of the Evidence Review Group Report}

The evidence regarding clinical effectiveness was based on three randomised controlled trials comparing the efficacy and safety of ixekizumab to placebo in patients with moderate-to-severe plaque psoriasis. In addition, the UNCOVER-2 and UNCOVER-3 studies included an active comparator (etanercept) arm. The data available indicate that ixekizumab is more efficacious in the treatment of moderate-to-severe plaque psoriasis in adults than placebo and etanercept. There were statistically significant increases in static Physician Global Assessment $(0,1)$ and PASI 75 response rates for patients treated with ixekizumab compared with placebo at week $12(p<0.001$ for all comparisons). Furthermore, the improvements in PASI response rate appeared to be maintained for up to 60 weeks during the long-term extension period. Health-related quality of life improved compared with baseline in significantly more patients with ixekizumab than with placebo and etanercept. The relative performance of ixekizumab in difficult-to-treat areas, including nails, scalp and palmoplantar areas, is broadly more efficacious than placebo and etanercept. However, the improvement of psoriasis symptoms of the face, which is included in the final scope, has not been reported in any of the UNCOVER studies. Ixekizumab was generally well tolerated in the UNCOVER trials.

It should be noted that the populations in the UNCOVER trials and the other studies used to inform the NMA were not fully in line with the final scope. In the CS, moderate-to-severe psoriasis was defined as a total PASI score of 10 or more and a DLQI score of more than 10 . However, the patients recruited in the UNCOVER trials were those with PASI scores of more than 12 and no restriction related to DLQI. The patients recruited in the NMA trials were not always those with PASI scores of 10 or more and their baseline DLQI scores were not clear.

The economic model described by the company is considered by the ERG to meet the NICE reference case for most part. The model structure is similar to models that 
were submitted in previous assessments and models described in the literature. Although common in this field, the ERG questions the use of relative PASI response to model the cost effectiveness as it may not reflect true differences in costs and health-related quality of life between treatments and treatment sequences. The model uses a treatment sequencing approach, which the ERG regards as superior to comparing single treatments. In the CS basecase analysis, it is assumed that treatment response does not depend on the position in the treatment sequence. Although it is suggested by clinical experts that this may be the case for treatment with different mechanisms of action, or when patients discontinue because of intolerance, this remains an area of uncertainty.

The company's base-case analysis resulted in an ICER of $£ 32,541$ per QALY gained (probabilistic results) for the ixekizumab sequence vs. the etanercept sequence. Other treatment sequences were dominated (secukinumab sequence) or extendedly dominated by the ixekizumab sequence. In the ERG base case, the sequence with ixekizumab as a second-line treatment after adalimumab (ADA-IXE) had an ICER of $£ 25,532$ per QALY gained vs. the etanercept sequence, and the ixekizumab in the firstline sequence had an ICER of $£ 39,129$ per QALY gained compared with ADA-IXE (probabilistic results). Explorative analyses showed that alternative assumptions regarding the population to derive utility estimates and costs of BSC were most influential.

\section{National Institute for Health and Care Excellence Guidance}

On April 26, 2017, NICE recommended ixekizumab as an option for treating adults with severe plaque psoriasis (i.e. PASI $\geq 10$ and DLQI $>10$ ) if the disease has not responded to standard systemic therapies, these therapies are contraindicated or the person cannot tolerate them. Moreover, ixekizumab treatment should be stopped after 12 weeks if the psoriasis has not responded adequately (i.e. 75\% reduction in the PASI score or a 50\% reduction in the PASI score in combination with a 5-point reduction in the DLQI score) and is authorised only when the company provides the discount agreed in the patient access scheme [3].

\subsection{Consideration of Clinical Effectiveness}

One issue discussed by the AC was that the trials included patients with a PASI score of 12 or more while previous appraisals of technologies for treating psoriasis defined a PASI score of 10 or more as severe disease. According to clinical experts, patients being considered for biological treatment tend to have a PASI score of 10,12 or more.
Therefore, the patients in the UNCOVER trials were representative of NHS patients considered for biological treatment. Furthermore, although previous treatment for trial participants varied, the populations of the UNCOVER trials were likely to be generalisable to patients in the NHS who would be considered for biological treatment. Overall, the $\mathrm{AC}$ concluded that the patients included in the UNCOVER trials were appropriate for decision making on the clinical effectiveness of ixekizumab [3].

The AC noted that the dose of etanercept administered in the trials was double that which is recommended in previous NICE's technology appraisal guidance (on etanercept and efalizumab for the treatment of adults with psoriasis) [11]. This dose was considered to be more effective than lower doses of etanercept, i.e. the odds ratios could underestimate the treatment effect of ixekizumab compared with etanercept. Nevertheless, the AC concluded that ixekizumab was more clinically effective than placebo and etanercept [3].

Three uncertainties related to the NMA were considered by the AC:

1. Trials of secukinumab that had not been included in the secukinumab appraisal [12] were part of the NMA.

2. It was uncertain how generalisable the results of the NMA were to ixekizumab being given as a first or second biological treatment in a sequence of biological treatments as a mixture of people who had and had not already had biological treatments were included in the trials

3. Results in the British Association of Dermatologists' Biologic Intervention Register [13] showed higher PASI scores for adalimumab compared with the CS.

The AC acknowledged that relative treatment effects rather than registry data are used in the NMA and that patients in the registry may differ from those included in the trials. Noting that the ERG highlighted the NMA results being in line with previous technology appraisals for psoriasis, the AC considered the NMA to be the more reliable source for decision making.

Based on the NMA, the AC concluded that ixekizumab was more clinically effective than adalimumab and ustekinumab, and agreed it was likely that ixekizumab was similarly effective compared with secukinumab and infliximab [3]. Regarding tolerability, the AC concluded that ixekizumab was similar to other biological treatments approved for treating psoriasis [3].

\subsection{Consideration of Cost Effectiveness}

The AC noted that the ERG considered the model structure, reflecting treatment sequences, to be better than comparing individual treatments as it more closely 
resembles clinical practice. The AC concluded that the company's model structure reflected clinical practice. The $\mathrm{AC}$ recognised that the treatment sequences presented did not cover all possible sequences but concluded that the sequences included by the company in its economic model reasonably represented current NHS practice.

The AC noted that the CS base-case incorporates utility gains during the maintenance period of treatment, but not during the induction period. Given that the AC heard (from both the ERG and the company) that ixekizumab is associated with a rapid response, it concluded that it would be appropriate to include utility gains for ixekizumab during the induction period (consistent with the ERG base case).

Although the AC considered the model structure, reflecting treatment sequences, to be reflective of clinical practice, it considered that including potentially non-cost effective comparators within sequences of treatments could result in misleading ICERs. Therefore, the AC considered the pair-wise comparisons of single-line biological therapy vs. BSC, which the ERG used to cross-validate the CS model, in its decision making. The AC noted that pairwise comparisons of the other biological treatments compared with BSC gave ICERs in the range of $£ 46,000-£ 74,000$ per QALY gained, while for ixekizumab this resulted in an ICER of $£ 41,000$ per QALY gained. The AC therefore concluded that the cost effectiveness of ixekizumab for treating moderate-to-severe plaque psoriasis was similar to that of other biological treatments, already recommended in previous NICE guidance, when compared with BSC. The committee concluded that the ICER was within the range that could be considered a cost-effective use of NHS resources.

\section{Key Methodological Issues}

In the plaque psoriasis disease area, PASI is the most used (primary) outcome in clinical trials. Consequently, it might be convenient to reflect this outcome in the model structure (i.e. data-driven model structure). There is however an important drawback associated with using PASI in the model structure. In health-state transition models, the health states are assumed to be homogeneous with regard to consequences for health and costs. When relative measures are used to define health states, this aspect may well be violated. Patients in a specific PASI relative response state may differ substantially with regard to health-related quality of life, further disease progression as well as resource consumption. For instance, PASI 75 corresponds to an improvement of $75 \%$ (i.e. $75 \%$ reduction in PASI score); this might entail going from a PASI score of 100 to 25 or from 40 to 10 . The possible implication of this approach is that the true impact of a treatment on quality of life and costs is not captured (e.g. given that the impact might be different for the two aforementioned examples) and hence results in biased cost-effectiveness estimates. The use of relative outcomes in the model structure is not restricted to the plaque psoriasis disease area; for instance, a 50\% reduction in seizure frequency is used in the field of epilepsy. Therefore, it is important to emphasise the limitations of this approach and ideally use alternative approaches.

Another issue related to the modelling approach is the use of treatment sequences. Although this approach might reflect clinical practice more closely compared with modelling single treatments, the amount of possible treatment combinations made it unfeasible to consider all potential treatment sequences. Hence, it is reasonable to compare a selection of treatment sequences, but selecting these sequences based on market share may not be the best option. Additionally, the AC also considered that this approach, including potentially non-cost-effective comparators within sequences of treatments, could result in misleading ICERs. This emphasizes the importance of carefully considering the treatment sequences being assessed.

Finally, the company programmed its model in the VBA code. The variables used in the VBA code were, however, not defined, nor linked to the CS. In addition, the VBA code contained multiple references requiring 'cell chasing' [14] to examine the implementation of the model. This severely hampered the transparency. Upon request by the ERG, the company provided a full list of the parameter names used in the Excel model, the VBA code and the description in the CS. This was helpful in gaining understanding of the technical implementation of the model. Nevertheless, owing to time constraints, the complexity and lack of transparency of the model, the ERG was unable to implement all analyses that it planned to implement. This illustrates the importance of transparency and documenting the technical implementation of the model to facilitate model scrutiny by external and/or internal reviewers.

\section{Conclusions}

This article describes the single technology appraisal considering ixekizumab for the treatment of moderate-to-severe plaque psoriasis. Although multiple methodological reservations can be made regarding the approach to estimate the cost effectiveness, the committee concluded that the ICER of ixekizumab vs. BSC was within the range that could be considered a cost-effective use of NHS resources. Therefore, ixekizumab was recommended as an option for treating plaque psoriasis in adults if (1) the disease is 
severe (PASI $\geq 10$ and DLQI $>10$ ); (2) the disease did not respond to standard systemic therapies (or these treatments are contraindicated) and; (3) the company provides ixekizumab with the agreed discount.

Acknowledgements The authors of this report would like to acknowledge Laura Coates who acted as a clinical advisor for this project. She commented on various clinical aspects but was not involved in the preparation of this paper. Moreover, we would like to acknowledge Ching-Yun Wei for her contribution to the health economics sections of the original ERG report.

Author Contributions All authors have commented on the submitted manuscript and have given their approval for the final version to be published. Robert Wolff, Gill Worthy and Jos Kleijnen reviewed the clinical-effectiveness evidence, and Caro Noake reviewed the search methods, Bram Ramaekers, Xavier Pouwels, Marije Oosterhoff, Anoukh van Giessen, Nigel Armstrong and Manuela Joore reviewed the cost effectiveness evidence. Bram Ramaekers acts as the overall guarantor for the manuscript. This summary has not been externally peer reviewed by Pharmacoeconomics.

\section{Compliance with Ethical Standards}

Funding This project was funded by the National Institute for Health Research Health Technology Assessment (HTA) Programme. See the HTA programme website for further project information (http://www. hta.ac.uk). This summary of the Evidence Review Group report was compiled after NICE issued the Final Appraisal Determination. The views and opinions expressed herein are those of the authors and do not necessarily reflect those of NICE or the Department of Health.

Conflict of interest Bram L.T. Ramaekers, Robert F. Wolff, Xavier Pouwels, Marije Oosterhoff, Anoukh Van Giessen, Gill Worthy, Caro Noake, Nigel Armstrong, Jos Kleijnen, and Manuela A. Joore have no conflicts directly relevant to the content of this article.

Open Access This article is distributed under the terms of the Creative Commons Attribution-NonCommercial 4.0 International License (http://creativecommons.org/licenses/by-nc/4.0/), which permits any noncommercial use, distribution, and reproduction in any medium, provided you give appropriate credit to the original author(s) and the source, provide a link to the Creative Commons license, and indicate if changes were made.

\section{References}

1. Eli Lilly and Company Limited. Ixekizumab for the treatment of moderate to severe plaque psoriasis [ID:904]: company submission to National Institute of Health and Care Excellence (Version 1.0). Single technology appraisal: Eli Lilly and Company Limited; July 2016. https://www.nice.org.uk/guidance/ta442/ documents/committee-papers-3. Accessed 11 Jun 2016.

2. Wolff R, Joore M, Pouwels X, Oosterhoff M, Ramaekers B, Van Giessen A, et al. Ixekizumab for treating moderate to severe chronic plaque psoriasis: a single technology appraisal. York: Kleijnen Systematic Reviews Ltd; 2016. https://www.nice.org. uk/guidance/ta442/documents/committee-papers-3. Accessed 3 Oct 2017.

3. National Institute for Health and Care Excellence. Ixekizumab for treating moderate to severe plaque psoriasis: final appraisal determination. London: National Institute for Health and Care Excellence; March 2017. https://www.nice.org.uk/guidance/ ta442/documents/final-appraisal-determination-document. Accessed 3 Oct 2017.

4. National Institute for Health and Care Excellence. Single technology appraisal. Ixekizumab for treating moderate to severe plaque psoriasis: final scope. London: National Institute for Health and Care Excellence; May 2016. https://www.nice.org.uk/ guidance/GID-TA10063/documents/final-scope. Accessed 6 Jun 2016.

5. European Medicines Agency. Summary of product characteristics (SmPC): Taltz, INN-ixekizumab. London: European Medicines Agency; 2016. http://www.ema.europa.eu/docs/en_GB/ document_library/EPAR_-_Product_Information/human/003943/ WC500205804.pdf. Accessed 9 Aug 2016.

6. Gordon KB, Blauvelt A, Papp KA, Langley RG, Luger T, Ohtsuki $\mathrm{M}$, et al. Phase 3 trials of ixekizumab in moderate-to-severe plaque psoriasis. N Engl J Med. 2016;375(4):345-56.

7. Griffiths CE, Reich K, Lebwohl M, van de Kerkhof P, Paul C, Menter A, et al. Comparison of ixekizumab with etanercept or placebo in moderate-to-severe psoriasis (UNCOVER-2 and UNCOVER-3): results from two phase 3 randomised trials. Lancet. 2015;386(9993):541-51.

8. Gordon KB, Langley RG, Leonardi C, Toth D, Menter MA, Kang $\mathrm{S}$, et al. Clinical response to adalimumab treatment in patients with moderate to severe psoriasis: double-blind, randomized controlled trial and open-label extension study. J Am Acad Dermatol. 2006;55(4):598-606.

9. Molnar FJ, Hutton B, Fergusson D. Does analysis using 'last observation carried forward' introduce bias in dementia research? CMAJ. 2008;179(8):751-3.

10. Lachin JM. Fallacies of last observation carried forward analyses. Clin Trials. 2016;13(2):161-8.

11. National Institute for Health and Care Excellence. Etanercept and efalizumab for the treatment of adults with psoriasis: NICE technology appraisal guidance 103. London: NICE; 2006. https:// www.nice.org.uk/guidance/ta103. Accessed 15 Mar 2016.

12. National Institute for Health and Care Excellence. Secukinumab for treating moderate to severe plaque psoriasis: NICE technology appraisal guidance 350. London: NICE; 2015. https://www. nice.org.uk/guidance/ta350. Accessed 15 Mar 2016.

13. Warren RB, Smith CH, Yiu ZZ, Ashcroft DM, Barker JN, Burden $\mathrm{AD}$, et al. Differential drug survival of biologic therapies for the treatment of psoriasis: a prospective observational cohort study from the British Association of Dermatologists Biologic Interventions Register (BADBIR). J Invest Dermatol. 2015;135(11):2632-40.

14. Tosh J, Wailoo A. NICE DSU: review of software for decision modelling. Sheffield: Decision Support Unit; 2008. https://www. ncbi.nlm.nih.gov/books/NBK425836/pdf/Bookshelf_NBK42583 6.pdf. Accessed 31 Oct 2017. 